\title{
Estrategias de marketing digital utilizadas por empresas del retail deportivo *
}

\author{
Digital Marketing Strategies Used by Sports Retail Companies \\ Sergio Luque-Ortiz (iD \\ Doctor en Comunicación, Universidad Europea Miguel de Cervantes, \\ Valladolid-España, sluque@uemc.es
}

\begin{abstract}
Cómo citar / How to cite
Luque-Ortiz, S. (2021). Estrategias de marketing digital utilizadas por empresas del retail deportivo. Revista CEA, v. 7, n. 13, e1650. https://doi.org/10.22430/24223182.1650
\end{abstract}

Recibido: 31 de agosto de 2020

Aceptado: 17 de noviembre de 2020

\begin{abstract}
Resumen
Con el fin de determinar qué tan efectivas son en la actualidad las estrategias de marketing en buscadores digitales utilizadas por empresas del retail deportivas, esta investigación ha tomado como caso de estudio las tiendas multideporte Decathlon y Sprinter por la importancia que ambas marcas tienen en el sector deportivo. La metodología empleada para llevar a cabo esta investigación, ha sido un análisis descriptivo mediante el estudio de diversos factores relacionados con el marketing digital, como son el posicionamiento en buscadores SEO (search engine optimization, por sus siglas en inglés), la usabilidad web y la creación de campañas de publicidad SEM (Search Engine Marketing, por sus siglas en inglés. La información se obtuvo usando diferentes herramientas empleadas en el marketing digital como Semrush y Screaming Frog. Se concluye que, a pesar de que ambas empresas realizan notables esfuerzos, los resultados son muy desiguales. Decathlon desarrolla estrategias de marketing digital más eficaces y útiles para la consolidación de la marca en términos de reputación y ventas, mientras que Sprinter presenta áreas con mejoras constantes.
\end{abstract}

Palabras clave: marketing digital, retail deportivo, estrategia mercadotécnica, plan de marketing, marketing deportivo.

Clasificación JEL: M30; M31.

\begin{abstract}
In order to determine the current effectiveness of the search engine marketing strategies used by sports retail companies, this paper examines the case of two multi-sport stores, Decathlon and Sprinter, due to the importance of both brands in the sports sector. The methodology implemented

*Este artículo se deriva del proyecto titulado «Estrategias de marketing digital utilizadas por empresas del retail deportivo» y ha sido financiado con recursos propios.
\end{abstract}


in this study was a descriptive analysis of several factors related to digital marketing, such as search engine optimization (SEO), website usability, and the creation of search engine marketing (SEM) campaigns. The information was collected using different tools widely employed in digital marketing, such as Semrush and Screaming Frog. It is concluded that, although both companies make remarkable efforts, their results are very dissimilar. Decathlon develops more efficient and useful digital marketing strategies to consolidate the brand in terms of reputation, while Sprinter presents areas for improvement.

Keywords: Digital marketing, sports retail, marketing strategy, marketing plan, sports marketing.

JEL classification: M30; M31.

\section{INTRODUCCIÓN}

El comercio electrónico se ha convertido en un canal de ventas esencial, sobre todo, desde finales de la década de 1990 (Elahi \& Hassanzadeh, 2009). En este sentido, según Martínez Martínez et al., (2007):

la aparición de la tecnología Internet trae consigo una nueva visión estratégica para el comercio detallista, generando nuevas oportunidades en el sector. El comercio electrónico supone la aparición de nuevas formas de comercialización en la cadena de distribución alimentaria, que operan complementando las formas de distribución tradicionales (p. 1).

Sobre lo dicho, Perdigón Llanes et al., (2018) establecen que:

la evolución de Internet potencia que el intercambio de informaciones se realice de manera más rápida y eficiente; además, posibilita que los usuarios accedan de una manera más fácil a bienes y servicios. Internet aumenta constantemente su nivel de penetración mundial, durante el 2017 el $51.8 \%$ de la población mundial era usuario de Internet, según indicadores del sitio Internet World Stats. El desarrollo tecnológico marca un cambio organizacional significativo en el sector empresarial mundial y potencia su participación en la era digital mediante las posibilidades que ofrece Internet y las TIC en el mundo de los negocios (p.192).

No hay duda de que el crecimiento y las posibilidades que ofrece Internet en la actualidad son prácticamente infinitas. Para Gangeshwer (2013)

la tecnología no solo ha ayudado a sobrepasar los límites geográficos existentes facilitando el intercambio de información entre compradores y vendedores, sino que también ha provocado el surgimiento de nuevos modelos de negocios entre los que destacan el comercio electrónico (p. 35).

Además de lo anterior, el comercio electrónico representa un proceso donde se produce un intercambio comercial. Por lo tanto, cabe recordar que las tiendas online tienen una importancia cada vez mayor y una mejor aceptación entre los consumidores españoles. Los datos así lo confirman.

Según un estudio sobre el comercio electrónico B2C (business to customer, o de la tienda al cliente, modalidad de la que se hablará con más detalle más adelante) elaborado por el ministerio de 
Economía de España en el año 2018 (actualizado con nuevos datos en la edición de 2019), en términos absolutos puede afirmarse que el volumen de negocio total del comercio electrónico en España alcanza los 41.509 millones de euros, lo que significa un $32.4 \%$ superior a los años anteriores. Del estudio analizado también se recoge otro dato revelador: el comercio electrónico está en expansión con una tasa de crecimiento anual por encima del 25\% (Observatorio Nacional de las Telecomunicaciones y de la Sociedad de la Información [ONTSI], 2019).

El informe señala que los datos de crecimiento se deben a varias razones. Por una parte, la mejora del contexto socioeconómico tras los efectos de la crisis financiera de 2008 en los hogares y en las empresas españolas; por otra, el creciente uso de Internet, y, sobre todo, el aumento de la confianza en las tiendas online por parte de los consumidores españoles (tradicionalmente muy reacios a este tipo de compra). De igual forma, según Paris et al., (2016) «el comercio electrónico ayuda a las organizaciones a realizar ahorros sustanciales de costos y aumentar los ingresos» (p. 45). Auspiciado y enriquecido por el marketing digital, Perdigón Llanes et al., (2018) reconocen que:

el empleo de Internet como fuente de publicidad y de difusión con el fin de aumentar las ventas de los productos ofertados (Lora y Segarra, 2013, p. 194) plantea que el marketing digital impulsa la creación de la demanda mediante el poder de Internet y constituye el proceso de comercialización de una marca a través de los canales digitales. Esta técnica de promoción representa un eslabón fundamental en la proliferación del comercio electrónico y en el desarrollo del e-business.

Por todo ello, podría afirmarse que, sin lugar a dudas, el e-commerce está en auge y requiere un análisis en profundidad en relación con las características distintivas de este tipo de comercio tan renovador en comparación al comercio tradicional (Cardona Valencia et al., 2019), sin olvidar las tipologías existentes, la descripción de los elementos clave a considerar en su gestión y la utilización de herramientas de marketing digital como recurso imprescindible para llegar al consumidor (ArangoBotero et al., 2020).

El desarrollo de las tiendas online no puede entenderse sin el nacimiento y posterior evolución de Internet (Goldman et al., 2020). En este sentido, la red de redes ha experimentado una progresión sin precedentes. Según el Diccionario de la Real Academia de la Lengua Española, [RAE] (2020), Internet es una red informática mundial, de carácter descentralizado, constituido por la conexión directa entre varias computadoras mediante la utilización de un protocolo especial de comunicación y conexión. En relación con este concepto y su nacimiento, Rodríguez Ávila (2017) apostilla que:

Internet fue creada a finales de los años 60 por el departamento de Defensa de los EEUU como red experimental llamada ARPANET (Advanced Research Projects Agency Network, Red de la Agencia de Proyectos de Investigación Avanzada). Su principal particularidad es que, en caso de ser dañado algún punto de ésta, no quedaría inactiva, garantizando así la imposibilidad de pérdida de la información. En el año 1971 se crea un software básico de envío y lectura de mensajes, y diez años más tarde se termina de definir el protocolo TCP/IP, que facilitará la comunicación entre los equipos informáticos. Se puede considerar que el nacimiento de Internet se produjo en el año 1983, cuando ARPANET se separó de la red militar en la cual tuvo su origen. Pero no es hasta 1991, cuando el CERN (Conseil Européen pour la Recherche Nucléaire, Consejo Europeo para la Investigación Nuclear) de Suiza lanza la WWW (World Wide Web, la telaraña mundial), y empieza el espectacular desarrollo experimentado por la red de redes. Dos años después nace la navegación al estar disponible el primer navegador web comercial, Mosaic, que permite el acceso de cualquier recurso disponible en Internet (p.10). 
Sobre lo anterior, la evolución de los buscadores de Internet tuvo lugar en paralelo a la extensión y posterior uso de Internet entre la sociedad. Así, en 1994 surge el primer navegador de uso comercial extendido: Yahoo! Posteriormente, en 1995, Microsoft lanza al mercado Internet Explorer, uno de los navegadores más conocidos de entorno Windows. Habría que esperar hasta 1998 cuando se produce el nacimiento de Google, y con él, la popularización del uso de los navegadores en la sociedad. En este sentido, para Suárez Sánchez-Ocaña (2012):

era el año 1998 cuando, casi sin esperarlo, se gestó el germen de lo que sería posteriormente el buscador más importante de internet y, más adelante, una de las empresas más poderosas e innovadoras del mundo. Sus creadores fueron dos veinteañeros, Sergey Brin y Larry Page [...] En aquella época internet era un mundo nuevo que había que descubrir. No estaba presente en nuestras vidas como lo está en la actualidad, aunque en las universidades, especialmente en Estados Unidos, su uso ya era habitual [...] Antes de la irrupción de Google los buscadores no eran un gran negocio y no se invertía demasiado en su desarrollo. Se concebían como herramientas secundarias, no como un elemento clave del desarrollo de la web. No representaban más que un servicio subalterno de los grandes portales de la época, como Yahoo! o Lycos (o como Terra en España), quienes pretendían ofrecer un servicio global en el que pasáramos buena parte de nuestro tiempo consumiendo publicidad. Aquellos portales eran un enorme cajón de sastre que incluía millones de páginas web mal interpretadas por los primarios robots de búsquedas que las almacenaban. [...] Desarrollar un método para clasificar la importancia de cada una de las páginas representaba un verdadero desafío. Fue así como Page y Brin comenzaron a desarrollar un algoritmo basado en fórmulas matemáticas para la búsqueda de datos [...] Aún no existía Google Inc. Como parte de su proyecto de investigación, Larry diseñó el software que rastreaba la red [...] En el otoño de 1997 decidieron que el motor de búsqueda que habían diseñado necesitaba otro nombre. Entre las posibilidades que barajaron se decidieron por Google (p. 102-103).

Así es como comienza la expansión de Google, no solo en la vida de millones de personas, sino también en las empresas. El desarrollo y posterior utilización de las estrategias de marketing online se produjo en paralelo a la transformación de Internet, dándose diferentes fases o etapas en el uso de las nuevas tecnologías. Precisamente, y según lo ya dicho, para Flores Diez (2015):

a lo largo del tiempo, el marketing ha ido evolucionando del Marketing 1.0 al Marketing 3.0. El marketing 1.0 consistía en hacer del producto el centro del sistema de producción. El siguiente nivel, el marketing 2.0, surge en la era de la información, las empresas intentan llegar al corazón y a la mente de sus consumidores y es por ello que la empresa debe segmentar, es decir, el centro es el consumidor. Ahora nos encontramos en el marketing 3.0, un tipo de marketing orientado al consumidor y que se pretende llevar a nivel espiritual (p. 52).

En relación a la evolución del marketing, Suárez-Cousillas (2018) no duda en señalar que se han dado cuatro etapas. La primera es el marketing 1.0. Éste surge en una época donde la producción en masa, tras la Revolución Industrial, estaba en pleno avance. Con un enfoque más centrado en la atención al cliente y a sus necesidades nace el marketing 2.0, muy unido por otra parte al Big Data. El marketing 3.0, por su parte, determina que los consumidores son seres humanos integrales y que sus necesidades siempre deben ser atendidas. Finalmente, en el marketing 4.0 el objetivo fundamental es la fidelización con el cliente y la confianza del mismo.

La irrupción del marketing digital, como una especialización del marketing convencional, conecta estrechamente con el entorno de Internet por lo que es necesario realizar una revisión bibliográfica 
completa en la que se analicen, por una parte, qué implica el concepto de e-commerce o tienda online y los tipos de tiendas existentes, junto a la puesta en marcha de estrategias de marketing digital (Taiminen \& Karjaluoto, 2015). Ante la redundancia de voces expertas que utilizan el concepto de marketing digital sin aportar una definición clara que sea capaz de englobar la complejidad del asunto, es necesario añadir una explicación sobre este término. Al respecto, y según Membiela-Pollán y Pedreira-Fernández (2019):

el marketing digital o e-marketing engloba buena parte de los usos propios de la esfera de los negocios. En concreto, comprende la utilización de Internet, las redes de telecomunicación y las tecnologías digitales relacionadas para conseguir los objetivos de marketing de la organización. Dicho de otro modo, es el conjunto de herramientas y estrategias digitales que ayudan a solucionar una necesidad de mercado generando beneficios (p. 3).

En relación a lo anterior, para Calle- Calderón et al., (2020):

al aplicar tecnologías digitales en una empresa mejoran la forma de comunicación con los interesados en adquirir el bien o el servicio, estas se basan en la aplicación de las $4 \mathrm{~F}$ (flujo, funcionalidad, feedback y fidelización), que son las variables que componen una estrategia de marketing efectiva, las cuales persiguen el único objetivo de transformar la forma de hacer negocios para brindar mayor comodidad a los consumidores, utilizando herramientas de comunicación más directa en donde la información se encuentra disponible en cualquier momento del día, gracias a la utilización de estos medios electrónicos capaces de realizar una evaluación acerca del comportamiento de los clientes (p. 343344).

Otros autores como Ballesteros López (2019) consideran que:

el marketing digital es el uso de Internet, dispositivos móviles, redes sociales y motores de búsqueda entre otros canales para llegar a los usuarios. En este sentido, el marketing digital representa una importante estrategia que proporciona a una persona u organización la capacidad de llegar a diferentes clientes a través del establecimiento de prácticas innovadoras (p. 110-111).

Por otra parte, es precisamente el aumento de la oferta y de la demanda de los productos o servicios lo que ha permitido que nuevas formas de hacer negocios proliferen (Abou-Elgheit, 2018). En este sentido, según Paris et al., (2016) «la aparición de nuevos negocios que rompen los esquemas tradicionales comerciales conocidos genera un entorno muy dinámico, en el que los consumidores encuentran lo que desean y las empresas ofrecen una disparidad de productos» (p. 76).

Una de las particularidades asociadas al marketing digital es que puede adherirse a cualquier empresa, sea del sector turístico y servicios, moda, restauración o educación. Al hilo de lo expresado, según Armijos-Delgado (2019):

las empresas necesitan de innovación e integración para ingresar en los mercados, con visión estratégica y herramientas que apoyen al desarrollo empresarial, las nuevas tecnologías que aportan al comercio electrónico, oportunidades con dinamismo y flexibilidad, son formas de establecer vías de comunicación con estrategias de uso de redes sociales y comunicación para conocimiento con los clientes (p. 2). 


\section{MARCO TEÓRICO}

En primer lugar, el concepto del comercio electrónico o e-commerce está en plena transformación, ya que los elementos que hacen posible su existencia (las TIC e Internet) también viven esa evolución de manera continuada (Cerqueus et al., 2020). De igual forma, para Anterportanlatinam Valero (2014):

para una definición de lo que es el comercio electrónico, se debe tener en cuenta que éste puede entenderse de diferentes formas, según la perspectiva que se adopte. Por lo tanto, dado su empleo en los ámbitos técnicos y de organización empresarial se puede abordar desde tres perspectivas principalmente: desde las comunicaciones, desde la de los procesos de negocio y la temporal (p. 11).

Por otra parte, en opinión de González López (2014):

conviven diferentes pareceres en relación al alcance directo del componente digital del e-commerce. Ello es debido a la falta de unanimidad en cuanto a la delimitación de qué se entiende por medios electrónicos, fruto del continuo progreso que se lleva a cabo en este ámbito del conocimiento (p. 25).

Asimismo, es necesario destacar que el interés por conocer más sobre la presencia de las tiendas online en las realidades empresariales y de los ciudadanos ha sido también objeto de estudio por parte de organismos oficiales. Es el caso de la Comisión Europea, que ya en 1997 realizó una primera aproximación sobre la existencia de tiendas online. En relación con lo anterior, Laudon y Traver (2014) no duda en señalar dos etapas diferenciadoras como son las siguientes:

\section{Etapa de invención}

Oscila desde 1995 hasta el año 2000. En esta primera etapa se produce la venta directa de productos en Internet con la incorporación de procesos transaccionales en determinados sitios web. De igual forma, se produce un crecimiento expansivo, sin precedentes y que influye hasta el punto de que los gobiernos y las autoridades políticas internacionales empiezan a ser conscientes de las notables posibilidades de Internet.

\section{Etapa de consolidación}

Engloba desde el año 2001 hasta el 2006. En este momento se produce una gran caída de ingresos por parte de múltiples e-commerce como consecuencia directa de la crisis. De igual forma, tiene lugar una corrección de los errores anteriores en lo que tiene que ver con la usabilidad del internauta, la presentación de los contenidos, etc. (p.23).

Herrera Acosta et al., (2019) señalan que los orígenes del comercio electrónico están en la década de los 60 en el momento en el que Electronic Data Interchange (EDI), considerado el primer sistema electrónico de intercambio de información, se instaura en diferentes empresas. Ya en la década de los 70, en concreto en 1979, se modifica de manera sobresaliente la comunicación con la instauración de Internet al crearse los dominios y los correos electrónicos de ámbito empresarial. De igual forma, en los 80 surgen las ventas por televisión que se verían reforzadas por el establecimiento de la venta 
online en la década de los 90 con el nacimiento de plataformas como Ebay o Amazon, entre otras. (p. 63).

Conocidas las fases principales que determinan la evolución de las tiendas online, es imprescindible describir cuáles son las tipologías existentes con la finalidad de encuadrar en las mismas los dos retails deportivos tomados como caso de estudio. Al hilo de lo expresado, se tomará como referencia el trabajo realizado por Varela Paz (2016). Según este autor, pueden darse diferentes categorías de tiendas online como son las siguientes.

\section{Business to customer (B2C)}

Es una relación comercial existente entre una empresa y el consumidor final. En la misma, según González López (2014) se produce un intercambio entre ambas partes. Las tiendas de Decathlon y Sprinter se enmarcan en este segmento.

\section{Business to customer (B2B)}

Es un tipo de comercio electrónico que engloba las transacciones y operaciones realizadas en Internet en las que una o dos empresas (fabricantes, distribuidores o negociadores finales) tienen relación entre sí.

\section{Consumer to consumer $(\mathrm{C} 2 \mathrm{C})$}

Esta tipología de las tiendas online se caracteriza por representar una transacción entre consumidores mediante la utilización y puesta en marcha de diversos proyectos que conectan a los clientes y a los proveedores de los productos (que no dejan de ser particulares). Ejemplos concretos son Ebay o Wallapop.

\section{Consumer to business (C2B)}

Este caso concreto de tienda online se caracteriza porque en el mismo no hay una auténtica respuesta del demandante ante una oferta concreta de la empresa vendedora, sino que es el propio cliente quien establece las condiciones específicas de la transacción. Algunos ejemplos son Privalia o Lets Bonus.

\section{Otros tipos de tiendas online}

Frente a las tipologías ya descritas también existen otro tipo de tiendas online en función a los agentes presentes en el proceso del negocio electrónico. Es el caso por ejemplo de las compras efectuadas entre empresas, consumidores y la Administración Pública.

En relación a lo ya descrito, es necesario añadir que la clasificación del comercio electrónico, según el tipo de transacciones, debe entenderse, al menos para Pesántez-Calva et al., (2020), como operaciones de comercio electrónico tales como B2B, B2C, C2B, G2B, G2C y G2G. 
Por otra parte, para que las tiendas online lleguen al consumidor final requieren de la realización de las estrategias de marketing digital. En este sentido, hoy en día las empresas cuentan con numerosas herramientas para la creación de las campañas de marketing online. Algunos de esos recursos, según Flores Diez (2015), son los siguientes:

\section{Estrategia SEO}

Tal y como señala Bizzocchi (s.f.), la optimización para motores de búsqueda (o SEO en inglés) comprende el trabajo y las estrategias que se realizan en un sitio web para que los motores de búsqueda detecten la información de la manera más adecuada posible, sin errores y sin elementos que interfieran en el proceso de posicionamiento orgánico o natural (p. 34).

El SEO, a su vez, se divide en dos tipos: SEO on y off page. El primero tiene que ver con factores relacionados con la calidad del contenido, la utilización de palabras clave o la creación de información novedosa. Por su parte, el segundo guarda una estrecha vinculación con elementos vinculados a la autoridad del dominio, la reputación, la interacción de los usuarios con el sitio web o el número de enlaces externos que genere el sitio web (p.24).

En relación con SEO, es necesario recordar que, según Iglesias-García y Codina (2016):

estas técnicas son diversas y cambiantes en función de la evolución de los buscadores. Por un lado, están las técnicas que implican la optimización de la página (con los llamados factores on page) y por otro, su socialización en Internet con otras páginas (los llamados factores off page) con la finalidad de mejorar la posición en los resultados de los buscadores a la hora de ofrecer los resultados de una búsqueda concretos. Estos factores son tenidos en cuenta por los buscadores a la hora de ofrecer los resultados de una búsqueda, en lo que se denomina Search Engine Results Page (más conocido como SERP por las siglas en inglés) (p. 932).

\section{Estrategia SEM}

En relación con SEO es imprescindible hablar del SEM. Para Bizzocchi (s.f.) «esta técnica consiste en la elaboración de campañas de pago para aumentar el número de visitas, ventas, clicks o contrataciones en una web. Este tipo de acciones garantizan que la empresa sea vista pero no garantizan que las visitas tengan una cierta continuidad en el tiempo como sí puede garantizarlo o lograrlo un trabajo continuado de posicionamiento orgánico en buscadores» (p. 35).

\section{Las redes sociales}

En el contexto actual de la comunicación y de la información multidireccional, las redes sociales son de gran importancia ya que garantizan mayores opciones de visibilidad para una empresa, y, sobre todo, un nuevo canal de comunicación con el cliente objetivo al que la empresa quiere llegar.

En relación a lo anterior, Kerpen (2012) señala que existen tantas redes sociales como perfiles de clientes, de manera que será oportuno segmentar qué plataformas sociales elegir en relación a la actividad de la empresa (p. 50). Además del trabajo referenciado de Flores Diez (2015) sobre las herramientas de marketing online más importantes para las empresas, existen más referencias. 
Autores como Membiela-Pollán y Pedreira-Fernández (2019) añaden la web corporativa y la tienda online, los blogs, las redes sociales (basadas en el perfil y en redes visuales), el email marketing, el SEO, el SEM, la publicidad digital y otras acciones.

\section{METOdOLOGÍA}

Desde el punto de vista metodológico se ha realizado una investigación de carácter descriptivo para abordar el estado de la cuestión del marketing digital de las empresas tomadas como estudio. Para ello se han analizado datos relativos al posicionamiento SEO, usabilidad web o UX y campañas de SEM de las empresas Decathlon y Sprinter. La información se ha obtenido utilizando herramientas empleadas en marketing digital como son las siguientes.

SEMrush: aplicación que proporciona datos sobre las búsquedas orgánicas, también informa de las búsquedas de pago, por lo que es de gran utilidad para estrategias SEM.

Screaming Frog: herramienta que rastrea diferente información estructurada de una web: el título, la descripción, el estado de cada una de las URL de la web y también las etiquetas del encabezado.

Tag Assistant: extensión gratuita de Chrome que ayuda a comprobar qué etiquetas de Google hay activas en una web.

Facebook Pixel Helper: extensión que permite saber si una web tiene configurado el píxel de Facebook para generar audiencias.

LikeAlyzer: herramienta que aporta información valiosa sobre perfiles de Facebook.

Google PageSpeed: sirve para medir a través de un test la velocidad de carga de una web.

Google Mobile Tester: útil para clasificar si un site es mobile friendly.

Las investigaciones descriptivas, según Viteri Luque et al., (2018):

buscan especificar las propiedades, características y los perfiles importantes de personas, empresas, grupos, comunidades o cualquier otro fenómeno que se someta a un análisis [...]. Los estudios descriptivos sobre técnicas de marketing son procesos basados en la búsqueda, recuperación, análisis, crítica e interpretación de datos secundarios, es decir, los obtenidos y registrados en fuentes documentales [...] Como en toda investigación, el propósito de este diseño es el aporte de nuevos conocimientos (p. 768-769).

De igual forma, en relación a las estrategias de marketing digital analizadas en las empresas tomadas como caso de estudio, se ha abordado el posicionamiento SEO desde una clasificación dual al reconocer elementos de SEO on y off page tal y como ya fueron mencionados en el apartado de marco teórico. En la Tabla 1 y Tabla 2, se adjuntan los principales elementos y características existentes de los mismos dentro de cada tipología de posicionamiento SEO. 
Tabla 1. Elementos de análisis SEO on page

Table 1. Elements of analysis in on-page SEO

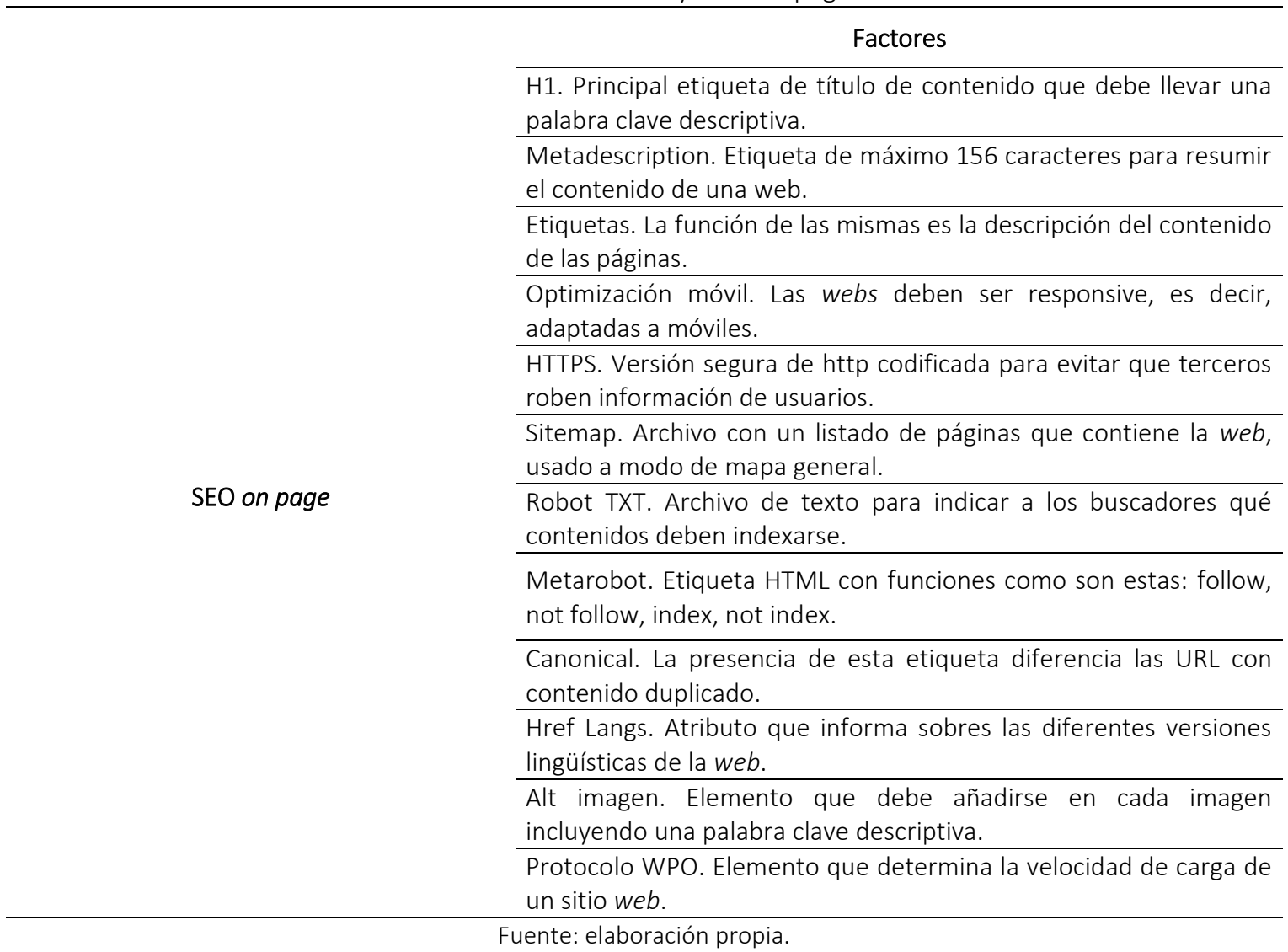

Con respecto a la optimización móvil, se tiene que, en el año 2015, Google emitió un comunicado en el que hacía oficial una práctica que ya se venía detectando entre los profesionales del marketing digital y expertos: el uso de la navegación web mediante teléfonos móviles y tablets cada vez era más creciente entre los usuarios. Ante esta situación, Google extendió la recomendación de incluir el diseño responsive (o diseño responsivo) en todas las webs realizadas (Más que Negocio, 2015).

Tabla 2. Elementos de análisis SEO off page

Table 2. Elements of analysis in off-page SEO.

\begin{tabular}{ll}
\hline \multirow{2}{*}{ SEO off page } & \multicolumn{1}{c}{ Factores } \\
\cline { 2 - 2 } & $\begin{array}{l}\text { Backlinks. Enlaces que apuntan desde otras webs a la propia. Son } \\
\text { links claves para el SEO. }\end{array}$ \\
\cline { 2 - 2 } & $\begin{array}{l}\text { Dominio de referencia. Es el dominio del que provienen los enlaces } \\
\text { externos que van al sitio. }\end{array}$ \\
\cline { 2 - 2 } & $\begin{array}{l}\text { Autoridad del dominio. Se calcula tras evaluar los factores } \\
\text { anteriormente descritos del sitio web. }\end{array}$ \\
\hline Fuente: elaboración propia.
\end{tabular}

Además de lo anterior, uno de los elementos más importantes que entran en relación en los sitios web es el de la usabilidad, es decir, la experiencia del usuario cuando efectúa una navegación en la 
página. En este sentido, se han medido diferentes atributos relativos a la usabilidad de las páginas analizadas y que se incluyen en la metodología efectuada. Los factores analizados de la UX se han escogido teniendo en consideración las aportaciones de Jackob Nielsen, un gurú del marketing digital que definió en 1995 los 10 principios básicos de usabilidad web y que, hoy por hoy siguen de plena actualidad y aplicación (ver Tabla 3). En estos se encuentran aspectos como la visibilidad del sitio web, la prevención de errores, la inclusión de elementos de información adicional, así como la seguridad que tiene el usuario en la navegación web cada vez que éste ingresa en el sitio (Arenzana, 2016).

Tabla 3. Elementos de análisis de la usabilidad del sitio web Table 3. Elements of analysis in website usability

\begin{tabular}{|c|c|}
\hline \multirow{9}{*}{ Usabilidad del sitio web } & Factores \\
\hline & $\begin{array}{l}\text { Información del producto. Los artículos mostrados deben } \\
\text { contener todos los detalles informativos relativos al mismo. }\end{array}$ \\
\hline & $\begin{array}{l}\text { Navegación interna. La optimización se realiza con un motor de } \\
\text { búsqueda propio que favorece una búsqueda rápida. }\end{array}$ \\
\hline & $\begin{array}{l}\text { Precio de los productos. Es importante que el precio del artículo } \\
\text { se muestre de manera clara y evidente. }\end{array}$ \\
\hline & $\begin{array}{l}\text { Valoraciones y reseñas de usuarios. Son un valor en alza ya que } \\
\text { aportan información objetiva ante los demás. }\end{array}$ \\
\hline & $\begin{array}{ll}\text { Personalización e identificación de los usuarios. La } \\
\text { personalización de los productos debe ser máxima. }\end{array}$ \\
\hline & $\begin{array}{l}\text { Facilidades de recogida de los productos. Las empresas optan por } \\
\text { el método clic and collect (recogida rápida). }\end{array}$ \\
\hline & $\begin{array}{l}\text { Transparencia en los métodos de envío y devolución. Deben } \\
\text { quedar claros los plazos y los motivos de devolución. }\end{array}$ \\
\hline & $\begin{array}{l}\text { Comunicación y atención al cliente. La comunicación debe ser } \\
\text { continuada con chatbox o asistentes virtuales al cliente. }\end{array}$ \\
\hline
\end{tabular}

Fuente: elaboración propia.

Finalmente, en lo que respecta a la metodología, también se han analizado las campañas de posicionamiento SEM o publicidad pagada en Google dispuesta por parte de los dos e-commerce estudiados en esta investigación. En las campañas de publicidad en Internet, y particularmente en Google, hay que diferenciar aspectos esenciales como son el tipo de campaña (red de búsqueda o red de display), el tipo de puja (manual o automática), además de la periodicidad de la información. Debido a que el análisis SEM conforma una parte concreta (que no la totalidad) del estudio descriptivo realizado, se han tomado como referencia algunos de los valores más relevantes de las campañas en relación a las empresas seleccionadas como son la inversión de capital efectuada en publicidad, la inclusión de palabras clave en los anuncios y la posición de media de los anuncios. En la Tabla 4 se incluyen más detalles al respecto. 
Tabla 4. Elementos de análisis de las campañas de SEM

Table 4. Elements of analysis in SEM campaigns

\begin{tabular}{|c|c|}
\hline \multirow{4}{*}{ Campañas de SEM } & Factores \\
\hline & $\begin{array}{l}\text { Inversión. La cantidad de dinero y el tipo de campaña efectuada } \\
\text { determinarán los resultados logrados en las webs. }\end{array}$ \\
\hline & $\begin{array}{l}\text { Palabras clave. Las keywords son fundamentales en una campaña de } \\
\text { SEM. La elección de las mismas depende de varios factores como el } \\
\text { tipo de puja (manual o automática), el tipo de concordancia (amplia } \\
\text { o exacta) y la audiencia. }\end{array}$ \\
\hline & $\begin{array}{l}\text { Posición media de los anuncios. Es el lugar en el que aparecen los } \\
\text { anuncios en relación con los anuncios de la competencia. La posición } \\
\text { estará determinada por varios factores como el tipo de puja realizada } \\
\text { por parte de las empresas. }\end{array}$ \\
\hline
\end{tabular}

Fuente: elaboración propia.

Junto a lo anterior, el estudio descriptivo se ha ejecutado con la información procedente de las herramientas de marketing digital ya mencionadas y que han permitido conocer con exactitud cada uno de los elementos explicados en los tres bloques de análisis incluidos en esta investigación, como han sido el posicionamiento SEO en buscadores, la usabilidad web y la presencia de campañas de publicidad SEM.

\section{RESULTADOS}

Una vez analizados los diferentes factores de posicionamiento SEO on y off page, la usabilidad del $e$ commerce y la creación de campañas SEM, se han procesado todos los datos. Para determinar el estado de los ítems evaluados, se ha seguido el mismo grado de valoración procedente de las herramientas de análisis SEO empleadas en la investigación. Al respecto, se ha aplicado una escala numérica repartida en tres bloques concretos como son del 10 al 8, del 8 al 5 y del 5 al 0 , y que a su vez representan un estado excelente, correcto o mejorable de los elementos abordados. De esta manera, se ha ofrecido un estudio objetivo y descriptivo basado en el estado de la cuestión del marketing digital de las empresas sometidas a investigación.

La Tabla 5, guarda una relación estrecha con el primero de los elementos sometidos a estudio en la investigación. Se trata del posicionamiento SEO a nivel on y off page de los sitios web analizados en el trabajo presentado. En este sentido se han abordado aspectos concretos como la inclusión o no de las etiquetas $\mathrm{H} 1$ o etiquetas de título en las páginas que componen los sitios web, la inclusión o no de metadescripciones en cada una de las páginas, la presencia o no del diseño responsive o adaptativo a móviles, además de la velocidad de carga en versión escritorio y en versión móvil, es decir el protocolo WPO, en los sitios analizados. 
Tabla 5. Resultado posicionamiento orgánico SEO on y off Page Decathlon y Sprinter Table 5. Scores of Decathlon and Sprinter in on-page and off-page SEO organic positioning

\begin{tabular}{|c|c|c|c|}
\hline \multirow{2}{*}{ Tipo de SEO } & \multirow{2}{*}{ Elementos SEO } & \multicolumn{2}{|c|}{ E-commerce } \\
\hline & & Decathlon & Sprinter \\
\hline \multirow{10}{*}{$\begin{array}{c}\text { SEO } \\
\text { on page }\end{array}$} & Robot y Sitemap & 10 & 10 \\
\hline & Https & 10 & 10 \\
\hline & Optimización móvil & 10 & 10 \\
\hline & Etiquetas $\mathrm{H} 1$ & 10 & 10 \\
\hline & Meta Descripción & 9,6 & 7.7 \\
\hline & Canonical & 9,9 & 9.2 \\
\hline & Hreflang & 10 & 10 \\
\hline & Imágenes & 10 & 10 \\
\hline & Velocidad de carga escritorio & 5.8 & 5 \\
\hline & Velocidad de carga móvil & 2,1 & 3.1 \\
\hline \multirow{2}{*}{ Tipo de SEO } & \multirow{2}{*}{ Elementos SEO } & \multicolumn{2}{|c|}{ E-commerce } \\
\hline & & Decathlon & Sprinter \\
\hline \multirow{3}{*}{$\begin{array}{c}\text { SEO } \\
\text { Off Page }\end{array}$} & Número de backlinks & 10 & 5 \\
\hline & Dominios de referencia & 10 & 6 \\
\hline & Puntuación de autoridad & 6.4 & 5 \\
\hline
\end{tabular}

Fuente: elaboración propia.

Ambos e-commerce obtienen datos muy similares en términos de posicionamiento SEO. En lo que respecta a los elementos on page, los valores extraídos son parecidos. De igual forma, puede verse que en ambas páginas la velocidad de carga en la versión móvil no está correctamente implementada por lo que debería realizarse una modificación del contenido añadiendo el protocolo WPO de carga rápida.

En cambio, las diferencias a nivel off page son más evidentes entre una página y otra. El número de backlinks que acumula la web de Decathlon es notablemente mayor en comparación a los resultados de Sprinter, obteniendo, además, dominios de referencia de mejor calidad. De esta primera unidad de análisis puede concluirse que, aunque los dos portales web tienen valoraciones correctas, Decathlon muestra un posicionamiento orgánico on y off page más sólido, lo que se traduce en un número mayor de visitas de internautas. En la Tabla 6 se muestran los datos recolectados en relación a la experiencia del usuario. 
Tabla 6. Resultados en relación a la usabilidad del e-commerce

Table 6. Scores of e-commerce usability

\begin{tabular}{|c|c|c|c|}
\hline \multirow{2}{*}{ UX web } & \multirow{2}{*}{ Factores } & \multicolumn{2}{|c|}{ E-commerce } \\
\hline & & Decathlon & Sprinter \\
\hline \multirow{9}{*}{$\begin{array}{c}\text { Elementos de } \\
\text { usabilidad }\end{array}$} & Información del producto & 10 & 5 \\
\hline & Navegación interna & 10 & 10 \\
\hline & Precio del producto & 10 & 5 \\
\hline & Valoraciones y reseñas de usuarios & 10 & 5 \\
\hline & $\begin{array}{l}\text { Personalización e identificación de } \\
\text { los usuarios }\end{array}$ & 10 & 10 \\
\hline & $\begin{array}{l}\text { Facilidades de recogida de los } \\
\text { productos }\end{array}$ & 10 & 0 \\
\hline & Métodos de envío & 10 & 10 \\
\hline & Política de devolución & 10 & 10 \\
\hline & Comunicación y atención al cliente & 8 & 9 \\
\hline
\end{tabular}

Fuente: elaboración propia.

Se han tomado en consideración diferentes variables como son la información del producto incluyendo el estocaje, las tallas disponibles, la descripción del artículo, el precio del producto, la forma de pago, los métodos de envío, además de incluir un análisis sobre la gestión comunicativa y de atención al cliente en lo que respecta a los sitios web con elementos como los chatboxs o asistentes virtuales, entre otros. Tras la aplicación de los diferentes ítems de análisis ya descritos se han obtenido datos determinantes. En primer lugar, la usabilidad web de ambos e-commerce es bastante elevada dándose valores muy altos en aspectos relevantes como la navegación interna, es decir, la inclusión de un buscador propio en el e-commerce, la personalización del usuario una vez que éste se identifica en el sitio web, además de la política de envíos. Estos son algunos de los valores en los que ambas empresas obtienen las mejores puntuaciones.

Sin embargo, existen diferencias en planteamientos de la usabilidad web que resultan importantes. Por ejemplo, la descripción e información del producto. Aunque ambas webs ofrecen una descripción de cada uno de los artículos, en el caso de la web de Decathlon la información añadida por cada producto es mayor incluyéndose aspectos adicionales como la guía de cuidados, las existencias y la inclusión de venta cruzada mediante las sugerencias de compras de otros usuarios.

Otro aspecto a valorar es la comunicación y la atención al cliente. Las dos webs muestran de manera clara los widgets o iconos de redes sociales promoviendo el tráfico web hacia estos canales. En el caso de Sprinter hay un paso más ya que incluso ofrece la posibilidad mediante la página web de enviar una petición para ser embajador de la marca.

Decathlon opta por una comunicación más tradicional usando diferentes métodos como son la atención telefónica o por correo. Uno de los aspectos que más diferencia genera entre una página y otra es la posibilidad de recoger los productos en la tienda, es decir, la utilización del sistema clic and collect. Mientras que Decathlon tiene implementado esta opción que facilita el ahorro del tiempo de 
los clientes, en el caso de Sprinter no se aprecia siendo un elemento negativo para la tienda online (ver Tabla 7).

Tabla 7. Resultados en relación a la creación de campañas de publicidad online SEM Table 7. Scores of online advertisement (SEM) campaigns

\begin{tabular}{cccc}
\hline \multirow{2}{*}{ SEM } & Elementos campana SEM & \multicolumn{2}{c}{ E-commerce } \\
\cline { 2 - 4 } Publicidad online & Volumen de inversión & Decathlon & Sprinter \\
\cline { 2 - 4 } & Palabra clave & 10 & 10 \\
\cline { 2 - 4 } & Posición media anuncios & 10 & 3 \\
\hline \multicolumn{2}{c}{ Fuente: elaboración propia. }
\end{tabular}

Se han abordado cuestiones muy concretas como son el volumen de inversión efectuado por los dos e-commerce analizados, la inclusión o no de palabras clave en los anuncios mostrados en Google, además de la posición media que obtienen los anuncios en las campañas de publicidad.

Sobre lo anterior, hay que tener en consideración que la investigación no se trata de analizar únicamente la publicidad digital mediante la realización de campañas SEM en e-commerce del sector retail deportivo, sino que éste es un factor más de análisis de los tres factores ya explicados en la fase metodológica.

Se han obtenido datos de interés en relación con la presencia de campañas de publicidad online en Google entre ambos e-commerce. Por una parte, el volumen de inversión de Sprinter es mayor en relación al de Decathlon. Sin embargo, recibe menos tráfico web que Decathlon. Según SEMrush, la web de Decathlon recibe solo un $8 \%$ del tráfico total mediante acciones de SEM, mientras que en el caso de Sprinter este porcentaje sube al 20\%. En este sentido, Decathlon realiza una inversión mensual de 78.000 euros en acciones de publicidad pagada. Sprinter invierte más 180.000 euros mensuales en Google Adwords, lo que tiene un efecto directo en las visitas que la página registra, equilibrando las visitas que no consigue de manera orgánica. Otro aspecto a considerar es el uso de las palabras clave, un factor determinante en las campañas de SEM. Las marcas deben hacer uso de las conocidas como branded keywords o palabras clave de empresa. Son términos que incluyen la marca de manera evidente. Para el portal SEMrush, la mayor parte del tráfico SEM que genera Decathlon procede de palabras clave de marca, lo que demuestra que los internautas acceden al sitio web tras visualizar los anuncios mostrados en Ads y que tienen un reconocimiento de la marca. Además, la marca utiliza mayor cantidad de palabras clave genéricas que ayudan a que las búsquedas tengan una visualización elevada cuando los internautas realizan las navegaciones.

En el caso inverso, a pesar de que Sprinter despliega todo un arsenal de medios económicos en las campañas de SEM, no incluye tantas palabras clave de marca lo que determina que, en parte, el posicionamiento de la marca no sea tan fuerte como el de su competidor principal Decathlon. Finalmente, la posición media que consiguen ambas marcas es sólida con la diferencia esencial de que Decathlon apoya el SEO en acciones de SEM dándose la situación contraria en el caso de Sprinter. Los resultados obtenidos muestran una información de interés en lo que respecta a la utilización de las estrategias de marketing digital para los e-commerce o las tiendas online del sector deportivo. Realizando una revisión de los mismos, y tras consultar otros estudios efectuados en la materia, se 
procede a explicar cuáles son las principales aportaciones y diferenciaciones de la investigación realizada.

En primer lugar, en el contexto actual de saturación de productos y servicios, cada vez es más difícil para las empresas captar la atención de los consumidores. En este sentido, es importante recordar que las estrategias de marketing digital pueden ayudar a que las empresas obtengan ese valor agregado o diferencial que tanto ansían.

En relación con lo anterior, las dos empresas tomadas como caso de estudio realizan esfuerzos y dedican recursos a la correcta implementación de las estrategias de marketing online. Sin embargo, los resultados son desiguales, o al menos, no son todo lo equitativos para lo que se esperaría en dos empresas del sector deportivo.

Por otra parte, es necesario destacar la principal aportación adicional que tiene esta investigación. En este sentido, no se han encontrado trabajos previos en los que se estudie de manera concreta y específica la utilización de las estrategias de marketing digital en las empresas del sector retail deportivo, tal y como se ha llevado a cabo en este trabajo presentado. Por lo tanto, la diferenciación obtenida es relevante, ya que esta investigación podría servir como documento de información y referencia bibliográfica para otros investigadores o autores.

Al respecto resulta sorprendente la escasez de trabajos específicamente realizados en este ámbito de estudio (marketing digital en relación a las empresas del sector deportivo) encontrándose un nutrido número de investigaciones que describen de manera general cuáles son las estrategias y herramientas de marketing digital usadas en la actualidad, además de estudios en los que se aborda el impacto publicitario de las marcas en los procesos de consumo sin que se haya hecho una vehiculización de ambos parámetros de estudio en un mismo trabajo.

\section{DISCUSIÓN}

La investigación presentada está influenciada, en cierta medida, por otros estudios ya existentes (en lo que se refiere a los trabajos vinculados al marketing digital y a las empresas de moda), aportando datos adicionales o novedosos. Algunos de los trabajos que se han cotejado para la realización de éste, y con los que se han podido encontrar ciertas similitudes, han sido realizados por Racionero Siles y Castillo Panadero (2015), Paz y Castillo (2017) y Pérez González et al., (2018).

En el caso de Racionero Siles y Castillo Panadero (2015), los dos investigadores realizaron un estudio en el que abordaron el análisis de las estrategias persuasivas de las grandes marcas deportivas centrando sus trabajos en diferentes indagaciones relativas a firmas bien posicionadas en el mercado internacional como son Nike, Adidas o Reebok.

Con relación al trabajo ejecutado por Paz y Castillo (2017), los autores ahondaron en las estrategias de marketing para la promoción de tiendas que venden bañadores femeninos. No hay que olvidar que, en las dos empresas seleccionadas para el trabajo presentado, los consumidores pueden comprar bañadores y otras prendas de tipología femenina. 
La investigación planteada por Pérez González et al., (2018) analiza la percepción de la personalidad de marca en artículos deportivos en estudiantes universitarios. En este trabajo, los autores toman como referencia diferentes empresas del sector deportivo entre, las que se encuentra Decathlon, pero con un enfoque de estudio no comparable con la investigación que se ha presentado.

Por lo tanto, las aportaciones y el valor añadido que tiene este trabajo son múltiples. Por una parte, la diferenciación en cuanto a los trabajos académicos publicados hasta el momento, siendo el primero que aborda de manera específica la relación entre marketing digital y empresas del retail deportivo; y de otro lado, la necesidad de incluir acciones de marketing digital en Decatlhon y Sprinter para que ambas empresas logren una posición sólida en el contexto online no resulta suficiente, máxime si se tiene en cuenta que la obtención de resultados definitivos requiere un tiempo de ejecución, aplicación y adaptación de las estrategias muy extenso.

Al respecto, las dos marcas están «obligadas» a invertir en marketing digital para conseguir una mayor reputación. Sin embargo, puede observarse como entre Decathlon y Sprinter hay diferencias clave. Mientras que Decathlon realiza una estrategia de SEO holística, atendiendo tanto a los factores on y off page y que redundan en un posicionamiento orgánico fuerte en Google, en el caso de Sprinter, éste retail solo atiende con especial interés al SEO on page, dejando de lado elementos de gran importancia para el posicionamiento.

En cuanto a la usabilidad web, puede verse cómo la estrategia Decathlon está basada en ofrecer una experiencia al usuario en la que la confianza, la seguridad y la atención al cliente están presentes en todo momento del proceso de compra del producto, mientras que en el caso de Sprinter la atención del cliente no está adaptada a las tendencias actuales del mercado que sí aplica Decathlon al no ofrecer aspectos esenciales como la posibilidad de realizar un pedido mediante la tienda online y ser recogido en cualquier tienda.

Finalmente, en lo que respecta a la inversión en publicidad, los datos hablan por sí solos. Decathlon realiza una acción de marketing basada en la confianza del cliente y en el posicionamiento orgánico en buscadores, frente a Sprinter que invierte más dinero en campañas de publicidad efímeras que garantizan una visibilidad elevada pero solo momentáneamente.

Por lo tanto, y como aspecto concluyente, puede afirmarse que la puesta en marcha de estrategias de marketing digital debe realizarse en diferentes parámetros que engloben tanto la presencia en buscadores con una aplicación de acciones que sirvan para reforzar aspectos de posicionamiento, sin olvidar la excelente atención al cliente al promover una exquisita usabilidad web y el diseño de campañas de publicidad puntuales para reforzar la imagen de marca y los beneficios.

\section{CONCLUSIONES}

Las nuevas tecnologías de comunicación han cambiado para siempre tanto la forma de comunicar como la manera en la que las empresas crean estrategias comerciales. Cada vez más, las empresas y los usuarios se están convirtiendo en creadores y consumidores de los servicios que ofrece Internet. Las corporaciones elaboran planes y acciones para destacar su oferta de artículos en un contexto saturado, mientras que los internautas hacen lo propio por adquirir nuevos y diferentes productos. 
En esta relación empresa-cliente, Google se ha convertido en el centro de unión de ambos elementos. Es el canal principal para lograr un posicionamiento orgánico o natural, que, reforzado con una estrategia de SEM, servirá para conseguir alcance, visibilidad e impacto para la adquisición de tráfico. En este sentido, los departamentos de marketing digital de los grandes retails del sector deportivo deben centrar sus esfuerzos en la presencia de buscadores, facilitando al algoritmo de Google el rastreo de los sitios web apareciendo en las SERP.

En este proceso se han encontrado algunas técnicas que han resultado satisfactorias para las empresas como tener perfectamente optimizados los factores básicos SEO, generar una red de backlinks con dominios de autoridad relevantes para la web y trabajar una estrategia SEM basada en términos de marca.

Todo este desarrollo de marca carece de sentido si no se cuida la usabilidad de la web del ecommerce. La relación de la experiencia del usuario con el éxito de su conversión es estrecha. Es necesario diseñar estratégicamente la forma en la que los usuarios interactúan con las distintas interfaces y fases de la compra y así obtener los resultados deseados de lealtad, ventas y fidelidad. Para finalizar, se establecen algunos puntos esenciales que resumen las conclusiones de la investigación. De igual forma, pueden extraerse algunas conclusiones relevantes.

La integración de los diferentes canales en una misma estrategia digital es necesaria. Una estrategia combinada de tráfico de pago en buscadores con campañas de posicionamiento SEO y la UX representan la mejor solución para lograr un posicionamiento de marca adecuado. La omnicanalidad es una tendencia afianzada en el comercio electrónico como se ha comprobado con las empresas abordadas.

La publicidad en Google ayuda a lograr tráfico inmediato procedente de usuarios de Internet, además de afinar las segmentaciones que mejoren la conversión y aumentar la visibilidad. La forma más rápida de conseguir tráfico SEM es con Google Ads. Para crecer de forma efectiva en términos de SEM es importante trabajar con una base presupuestaria media o alta que garantice una correcta consecución de objetivos, además de tener muy claro que las acciones de SEM deben ser un apoyo puntual al resto de acciones de la estrategia de marketing online.

La suma de todos los factores anteriormente descritos determina el éxito en las estrategias de marketing digital. La creación de una estrategia de marketing digital es compleja. Supone tiempo, esfuerzo y dinero, tanto la elaboración de un plan de acción en el que se incluyan diferentes fases de actuación, como la espera hasta ver resultados.

En el caso de los e-commerce analizados, aunque las dos empresas del sector retail deportivo ofrecen resultados positivos de posicionamiento SEO y usabilidad web, se han observado diferencias que pueden deberse a la implementación de un conjunto de acciones globales frente a una serie de actuaciones concretas. En el entorno actual es imprescindible que las empresas ejecuten planes completos, adaptados a las tendencias actuales del mercado, pero también a las necesidades de los clientes de manera que cada vez más el marketing online facilite el día a día de los consumidores. 


\section{CONFLICTOS DE INTERÉS}

El autor declara que no presenta conflictos de interés financiero, profesional o personal que pueda influir de forma inapropiada en los resultados obtenidos o las interpretaciones propuestas.

\section{REFERENCIAS}

Abou-Elgheit, E. (2018). Understanding Egypt's emerging social shoppers. Middle East Journal of Management, v. 5, n. 3, 207-270. https://doi.org/10.1504/MEJM.2018.093611

Anterportanlatinam Valero, J. M. (2014). Relevancia del e-commerce para la empresa actual (Trabajo de grado). $\underline{U R L}$

Arango-Botero, D.; Valencia-Arias, A.; Bermúdez-Hernández, J.; Duque Cano, L. (2020). Factors that promote social media marketing in retail companies. Contaduría y Administración, v. 66, n. 1, e233. http://dx.doi.org/10.22201/fca.24488410e.2021.2475

Arenzana, D. (2016). Principios de usabilidad web de Jackob Nielsen: diseño UX. URL

Armijos-Delgado, N. M. (2019). Marketing digital: una herramienta para potenciar y promocionar las mypimes en el ámbito internacional. Innova Research Journal, v. 4, n. 1.

https://doi.org/10.33890/innova.v4.n1.2019.764

Ballesteros López, L. G.; Silva Ordoñez, I. F.; Mena Mera, D. J.; Angamarca Pillajo, M. E. (2019). Estrategias de marketing digital en empresas ecommerce: un acercamiento a la perspectiva del consumidor. 593 Digital Publisher CEIT, v. 4, n. 5-1, 108-122. https://doi.org/10.33386/593dp.2019.5-1.123

Bizzocchi, A. (s.f.). SEM vs. SEO: ¿Cuáles son las diferencias? $\underline{\mathrm{URL}}$

Calle-Calderón, K. C.; Erazo-Álvarez, J. C.; Narváez-Zurita C. I. (2020). Marketing digital y estrategias online en el sector de fabricación de muebles de madera. Revista Arbitrada Interdisciplinaria Koinonía, v. 5, n. 10, 339-369. http://dx.doi.org/10.35381/r.k.v5i10.698

Cardona Valencia, D.; Valencia-Arias, A.; Bran, L.; Benjumea, M.; Valencia-Arias, J. (2019). Analysis of e-commerce acceptance using the technology acceptance model. Scientific Papers of the University of Pardubice. Series D. Faculty of Economics and Administration, n. 45, 174-185. URL

Cerqueus, T.; Bonnaud, J.; Dashkov, O.; Morin, E. (2020). Thesaurus matching in electronic commerce. Electronic Commerce Research, 1-26. https://doi.org/10.1007/s10660-020-09438-9

Elahi, S.; Hassanzadeh, A. (2009). A framework for evaluating electronic commerce adoption in Iranian companies. International Journal of Information Management, v. 29, n. 1, 27-36. https://doi.org/10.1016/j.ijinfomgt.2008.04.009 
Flores Diez, A. (2015). Análisis del comercio electrónico en España (trabajo de grado). URL

Gangeshwers, D. K. (2013). E-commerce or Internet Marketing. A Business Review from Indian Context. International Journal of u-and e-Service, Science and Technology, v. 6, n. 6, 187-194. http://dx.doi.org/10.14257/ijunesst.2013.6.6.17

Goldman, S. P.; van Herk, H.; Verhagen, T.; Weltevreden, J. W. (2020). Strategic orientations and digital marketing tactics in cross-border e-commerce: Comparing developed and emerging markets. International Small Business Journal. https://doi.org/10.1177/0266242620962658

González López, O. R. (2014). Comercio electrónico 2.0. Anaya Multimedia.

Herrera Acosta, J. F.; Vásquez Torres, M. C.; Jacobo Hernández, C. A. (2019). Tendencias de compra en el comercio electrónico por estudiantes universitarios; un caso de estudio. 593 Digital Publisher CEIT, v. 4, n. 6, 61-73. https://doi.org/10.33386/593dp.2019.6.136

Iglesias-García, M.; Codina, L. (2016). Los cibermedios y la importancia estratégica del posicionamiento en buscadores (SEO). Opción Revista de Ciencias Humanas y Sociales, v. 32, n. especial 9, 929-944. URL

Kerpen, D. (2012). Me gusta: conseguir el éxito en las redes sociales. Anaya Multimedia.

Laudon, K. C.; Traver, C. G. (2014). E-Commerce (10 Ed.). Pearson Prentice Hall.

Martínez Martínez, M.; Saco Vázquez, M.; Fernández Rodríguez, R. (2007). Desarrollo y evolución del comercio electrónico en la distribución alimentaria minorista. En C. Mercado (Coordinador), Empresa global y mercados locales. XXI Congreso Anual AEDEM Universidad Rey Juan Carlos, Madrid, España. URL

Más que Negocio (2015). Google eliminará las webs sin diseño responsive a partir del 21 de abril. URL

Membiela-Pollán, M. E.; Pedreira-Fernández, N. (2019). Herramientas de Marketing digital y competencia: una aproximación al estado de la cuestión. Atlantic Review Of Economics - AROEC, v. 3, n. 3. URL

Observatorio Nacional de las Telecomunicaciones y de la Sociedad de la Información (2019). El comercio electrónico B2C en España 2018. ONTSI. https://doi.org/10.30923/2172-458x/b2c/201911

Paris, D. L.; Bahari, M.; lahad, N. A.; Ismail, W. (2016). Systematic literature review of E-commerce implementation studies. Journal of Theoretical and Applied Information Technology, v. 89, n. 2, 422-438. $\underline{U R L}$

Paz, M. y Castillo, E. (2017). Estrategias de marketing digital para la promoción en tiendas de trajes de baño femenino. Marketing Visionario, v. 6, n. 1, 96-115. URL 
Perdigón Llanes, R.; Viltres Sala, H; Madrigal Leiva, I. R. (2018). Estrategias de comercio electrónico y marketing digital para pequeñas y medianas empresas. Revista Cubana de Ciencias Informáticas, v. 12, n. $3,192-208$. URL

Pérez González, B.; Burillo, P.; Sánchez Burón, A. (2018). Percepción de la personalidad de marca en artículos deportivos en estudiantes universitarios españoles. SPORT TK-Revista EuroAmericana de Ciencias del Deporte, v. 7, n. 1, 71-80. https://doi.org/10.6018/321911

Pesántez-Calva, A. E.; Romero-Correa, J. A.; González-Illescas, M. L. (2020). Comercio electrónico B2B como estrategia competitiva en el comercio internacional: desafíos para Ecuador. Innova Research Journal, v. 5, n. 1, 72-93. https://doi.org/10.33890/innova.v5.n1.2020.1166

Racionero Siles, F.; Castillo Panadero, J. L. (2015). Publicidad y deporte: análisis de las estrategias persuasivas de las grandes marcas deportivas. Lecturas: Educación Física y Deportes, Revista Digital, n. 204. URL

Real Academia Española (19 de noviembre del 2020). Diccionario de la lengua española (23 ed.). [Versión 23.3 en línea]. URL

Rodríguez Ávila, A. (2017). Iniciación a la red Internet: concepto, funcionamiento, servicios y aplicaciones de Internet. Ideaspropias Editorial.

Suárez -Cousillas, T. (2018). Evolución del marketing 1.0 al 4.0. Redmarka Revista de Marketing Aplicado, v. 1, n. 22, 209-227. https://doi.org/10.17979/redma.2018.01.022.4943

Suárez Sánchez-Ocaña, A. (2012). Desnudando a Google: nacimiento de un imperio. Derecom, n. 10, 99-115. URL

Taiminen, H.; Karjaluoto, H. (2015). The usage of digital marketing channels in SMEs. Journal of Small Business and Enterprise Development, v. 22, n. 4, 633-651.

https://doi.org/10.1108/JSBED-05-2013-0073

Varela Paz, D. (2016). El comercio electrónico en la economía española: Tipología, marco legal, principales magnitudes y tendencias (Trabajo de grado). $\underline{U R L}$

Viteri Luque, F. E.; Herrera Lozano, L. A.; Bazurto Quiroz, A. F. (2018). Importancia de las técnicas de marketing digital. RECIMUNDO Revista Científica de la investigación y el conocimiento, v. 2, n. 1, 764-783. URL 